\title{
Review of: "Quiescin sulfhydryl oxidase 1 promotes sorafenib-induced ferroptosis in hepatocellular carcinoma by driving EGFR endosomal trafficking and inhibiting NRF2 activation"
}

Ying Wang

Potential competing interests: The author(s) declared that no potential competing interests exist.

Review of: Quiescin sulfhydryl oxidase 1 promotes sorafenib-induced ferroptosis in hepatocellular carcinoma by driving EGFR endosomal trafficking and inhibiting NRF2 activation

Ying Wang ${ }^{1}$

1 Clinical Research Center, Zhejiang Provincial People's Hospital, 310014, China

As a fundamental research, this study found that Quiescin sulfhydryl oxidase 1 (QSOX1), acting as a cellular pro-oxidant, disrupted redox homoeostasis and sensitizes HCC cell to oxidative stress. The underlying mechanism is QSOX1 inhibit NRF2 activation though interacts with EGFR to enhance its ligandinduced endosomal transfer and lysosomal degradation. Therefore, this study suggests that QSOX1 can cooperate with sorafenib leading to enhanced ferroptosis of HCC cells. In general, it is a well-performed study which firstly showed the unreported function in redox regulation of QSOX1.

However, there are a number of concerns with this study which include the following:

1. In the whole study, the authors used QSOXI overexpression in MHCC97H cell line and shQSOX1 in Hep3B cell line. Since different cell line has different gene/protein expression profile, features, drug sensitivity... For example, MHCC97H is a human hepatocellular carcinoma with highly metastasis potential, while Hep3B is a primary human hepatocellular carcinoma with low metastasis potential. As shown in Figlc, the GSH/GSSG level in MHCC97H (vector) is almost 7.5, however in Hep3B (shNT) is 3, suggesting a different basal level in different cell lines. Thus, both the overexpression and knock down needed to be verified in more than 2 cell lines are necessary. And the authors should explain why MHCC97H and Hep3B were chosen in this study.

2. We have noticed that the relationship between tumor QSOX1 and NRF2 levels was validated in a cohort of tumor tissues taken from patients with hepatitis B-related HCC. In addition, both MHCC97H and Hep3B cell lines contain hepatitis B virus gene. Therefore, are the authors mainly focused on hepatitis B-related HCC, which is especially universal in China but not worldwide. In addition, the authors focused on Sorafenib's effect on HCC though ferroptosis and used Sorafenib as a ferroptosis inducer in this study, 
however due to the different effect of Sorafenib on HBV-derived HCC or non HBV-derived HCC, it is necessary to discuss the role of QSOX1 on different HCC types.

3. In Fig3f, Kaplan-Meier analyses for overall survival (OS) was per[1]formed on the $151 \mathrm{HCC}$ patient samples using the subgroup classifica[1]tion, however, we are interested in the sample size in each subgroup. 